\title{
Fluorescence resonance energy transfer from tryptophan in human serum albumin to a bioactive indoloquinolizine system
}

\author{
PARAMITA DAS, ARABINDA MALLICK,* BASUDEB HALDAR, ALOK \\ CHAKRABARTY and NITIN CHATTOPADHYAY* \\ Department of Chemistry, Jadavpur University, Kolkata 700032 \\ e-mail: pcnitin@yahoo.com
}

\begin{abstract}
The interaction between a bioactive molecule, 3-acetyl-4-oxo-6,7-dihydro-12H indolo-[2,3-a] quinolizine (AODIQ), with human serum albumin (HSA) has been studied using steady-state absorption and fluorescence techniques. A $1: 1$ complex formation has been established and the binding constant $(K)$ and free energy change for the process have been reported. The AODIQ-HSA complex results in fluorescence resonance energy transfer (FRET) from the tryptophan moiety of HSA to the probe. The critical energy-transfer distance $\left(R_{0}\right)$ for FRET and the Stern-Volmer constant $\left(K_{\mathrm{sv}}\right)$ for the fluorescence quenching of the donor in the presence of the acceptor have been determined. Importantly, $K_{\mathrm{SV}}$ has been shown to be equal to the binding constant itself, implying that the fluorescence quenching arises only from the FRET process. The study suggests that the donor and the acceptor are bound to the same protein at different locations but within the quenching distance.
\end{abstract}

Keywords. Fluorescence resonance energy transfer; photosensitisation; human serum albumin; fluorescence quenching; overlap integral; Stern-Volmer constant.

\section{Introduction}

Fluorescence resonance energy transfer $\left(\right.$ FRET) ${ }^{1,2}$ is a distance-dependent interaction between the different electronic excited states of dye molecules in which excitation energy is transferred from one molecule (donor) to another molecule (acceptor) at the cost of the emission from the former molecular system. The process is also known as Förster's resonance energy transfer (FRET). It is a powerful physical and/or biophysical technique that permits analysis of the interaction between the fluorophores in proximity. FRET is an interaction that depends largely on the distance between the interacting partners. However, it is not the only factor influencing the efficiency of the energy transfer process. According to Förster's theory, the efficiency of FRET depends principally on the following factors: (i) the extent of overlap between the donor emission and the acceptor absorption, (ii) the orientations of the transition dipoles of the donor and the acceptor, and (iii) the distance between the donor and the acceptor. ${ }^{1,2}$ The lifetime of a fluorophore in the excited state is normally in the range of nanoseconds. After excitation, competition prevails between the various decay processes, namely,

*For correspondence fluorescence and non-radiative deactivations, including energy transfer to the surrounding molecules. Efficiency of FRET is known to depend on the inverse sixth power of the intermolecular distance between the donor and the acceptor molecules. ${ }^{1}$ Because of the high sensitivity to the proximities of the interacting units in the nanometre range, people often use this technique for mapping cell surface parameters. ${ }^{3}$ The most widespread application of this phenomenon is its use as a spectroscopic ruler. ${ }^{1}$ Most of the applications of the FRET phenomenon are in the field of biochemistry. It is an important technique for investigating a variety of biological phenomena including energy transfer processes. ${ }^{1,4,5}$ One important consequence of such energy transfer is photosensitization, a classic example of which is photosynthesis. ${ }^{2}$ Further, FRET plays a key role in the photodynamic therapy (PDT) in cancer treatment. ${ }^{1,2}$ It is also used extensively to study the structure, conformation, spatial distribution and assembly of complex proteins. ${ }^{5}$ The technique has also been exploited to locate the positioning of a fluorophore attached to a DNA. ${ }^{6}$ Since FRET is involved in a number of biological phenomena and/or used to study biological photoprocesses, interest has been paid to the studies of FRET from many fluorophores of biological interest in vitro. 
Human serum albumin (HSA) and bovine serum albumin (BSA), abundant in plasma, are the most widely studied transport proteins. The primary structure of HSA has about 580 amino acid residues and is characterized by low tryptophan content and high cystine content, stabilizing a series of nine loops. The secondary structure of this protein comprises $67 \%$ of a helix of six turns and 17 disulphide bridges. $^{7,8}$ The tertiary structure is composed of three domains I, II and III. Each domain is constituted by a cylinder formed by six helices, and each one of these domains is constituted by two subdomains formed by three helices that are covalently linked by a double-cystine bridge. These subdomains are named IA, IB, IIA, IIB, IIIA and IIIB ${ }^{7,8}$ Since domains II and III share a common interface, binding of a probe to domain III leads to conformational changes affecting binding affinities to domain II. The tryptophan residue plays an important structural role in the formation of the IIA binding site by limiting solvent accessibility. The molecular weight of HSA is $66.5 \mathrm{kD}{ }^{8}$ HSA contains only one tryptophan residue (Trip-214). This tryptophan participates in additional hydrophobic packing interactions at the IIA-IIIA interface. ${ }^{7,8}$

It is well known that reactive oxygen species (ROS) participate in a number of pathological processes in the nervous system. ${ }^{9}$ Compounds able to interfere with the action of ROS might be useful in prevention and treatment of these pathologies. ${ }^{10}$ Recently, attention has been focussed toward designing and synthesizing compounds with a suitable spectrum of pharmacological and pharmaco-kinetic properties, among which indole derivatives are a distinct group with great potential. The indole nucleus seems to be a promising basic unit for designing and synthesis of new derivatives able to protect the nervous system. ${ }^{11}$ Molecules containing the indole nucleus like $\beta$ carbolines, carbazoles etc. are by now well established as bioactive molecules. ${ }^{12-16}$ The single-step synthesis of 3-acetyl-4-oxo-6,7-dihydro-12H indolo[2,3-a] quinolizine (AODIQ) from 1-methyl-3,4dihydro- $\beta$-carboline leads to the assumption that the molecule should have biological activity. ${ }^{17}$ Through a series of experiments we have already established that the fluorophore molecule serves as an excellent fluorescent probe for biological systems. ${ }^{18-23}$ Given the broad range of biological activities of AODIQ, in the present study we have explored the FRET process operative between the tryptophan residue present in HSA and the AODIQ system in some detail.

\section{Experimental}

3-Acetyl-4-oxo-6,7-dihydro-12H indolo-[2,3-a] quinolizine (AODIQ) (figure 1) was synthesized in the laboratory using the method described earlier. ${ }^{17}$ It was purified by column chromatography and the purity of the product was checked by thin layer chromatography (TLC). The compound was further vacuum-sublimed before use. Triply distilled water was used for making the experimental solutions. HSA and HEPES buffer were purchased from Sigma-Aldrich, USA; $50 \mathrm{mM}$ HEPES buffer solution was prepared in water and the $\mathrm{pH}$ was adjusted to 7.0 and was used in all the studies of AODIQ in protein. The absorption and fluorescence studies were performed using a Shimadzu MPS 2000 spectrophotometer and a Spex Fluorolog II spectrofluorimeter respectively.

\section{Results and discussion}

A prerequisite of a meaningful FRET investigation in a protein-probe system needs establishment of the stoichiometry of the protein:probe complex. Before going into the study of FRET we followed the fluorescence behaviour of AODIQ with the addition of HSA in buffered aqueous medium (figure 2) to affirm the composition of the HSA-AODIQ complex. There was no visible change in the absorption spectrum of AODIQ upon addition of HSA. On the contrary, there was a phenomenal change in the fluorescence profile. The broad emission band of the fluorophore showed a blue shift with an enhancement in the fluorescence yield. It was also associated with a narrowing in the full width half maximum (FWHM) of the emission band. The remarkable modification in fluorescence behaviour clearly indicates interaction between fluorophore and protein.

The binding interaction was studied using the double reciprocal plot following the BenesiHildebrand equation, ${ }^{24}$

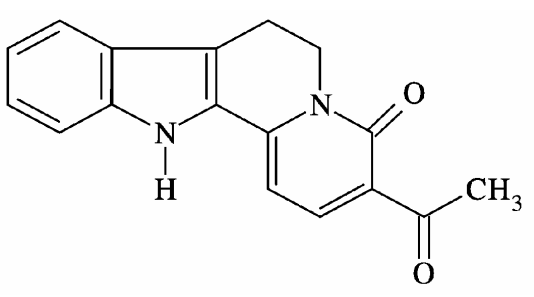

Figure 1. Structure of AODIQ. 


$$
\frac{1}{F_{x}-F_{0}}=\frac{1}{F_{\infty}-F_{0}}+\frac{1}{K\left(F_{\infty}-F_{0}\right)[\mathrm{HSA}]},
$$

where $F_{0}, F_{x}$ and $F_{\infty}$ are the integrated fluorescence intensities (areas under the fluorescence curves) of AODIQ considered in the absence of protein, at an intermediate protein concentration, and at a concentration of complete interaction, respectively; $K$ being the binding constant and [HSA] the protein concentration.

A plot of $\left(F_{x}-F_{0}\right)^{-1}$ against $[\mathrm{HSA}]^{-1}$ shows linear variation (figure 3 ) justifying the validity of the

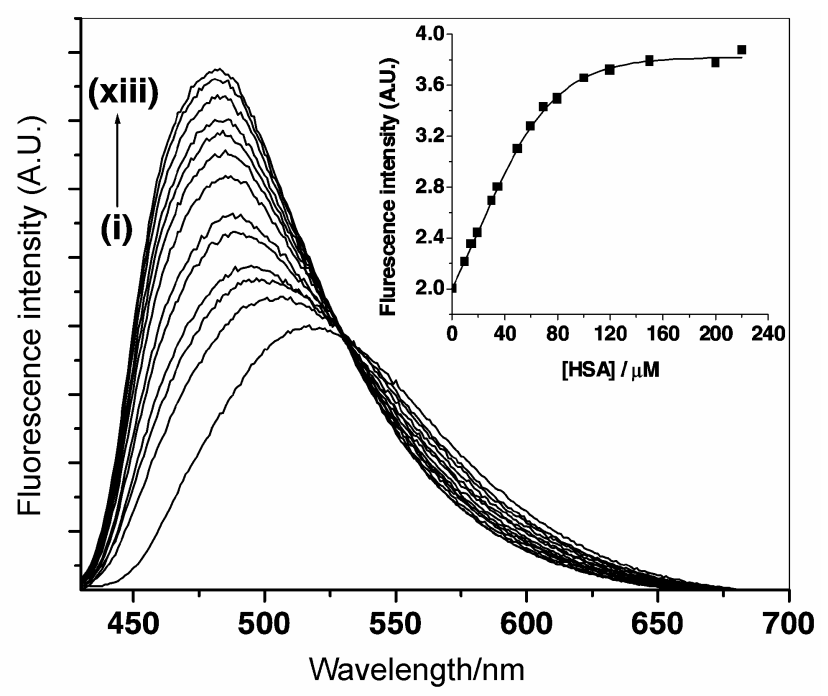

Figure 2. Emission spectra of AODIQ as a function of HSA concentration $\left(\lambda_{\text {exc }}=420 \mathrm{~nm}\right)$. Curves (i) $\rightarrow$ (xiii) correspond to $0,10,15,20,30,35,50,60,70,80,100$, $150,220 \mu \mathrm{M}$ HSA respectively. Inset shows the variation of fluorescence intensity as a function of HSA concentrations.

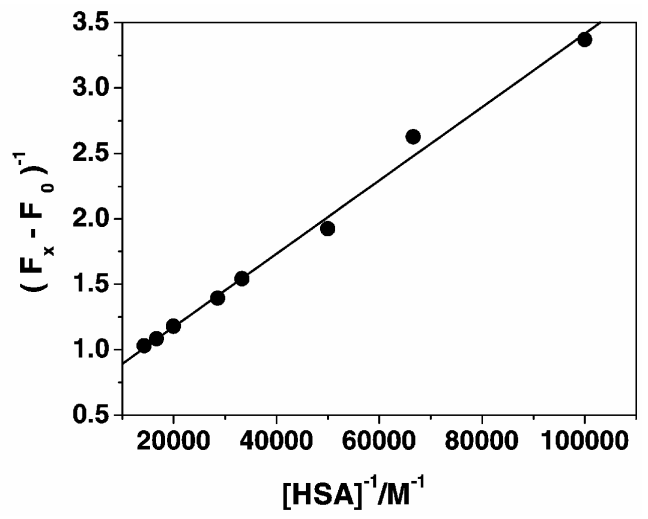

Figure 3. Double reciprocal plot for the complexation between AODIQ and HSA.
Benesi-Hildebrand equation for the present case and hence confirms one-to-one stoichiometry for the probe: HSA complex. From the ratio of intercept to slope of the plot the binding constant has been determined to be $2 \cdot 1( \pm 0 \cdot 2) \times 10^{4} \mathrm{~mol}^{-1} \mathrm{dm}^{3}$; which corresponds to a value of $-24.9 \mathrm{~kJ} \mathrm{~mol}^{-1} \mathrm{dm}^{3}$ for the free energy change $(\Delta G)$ of the complexation process.

The tryptophan moiety present in HSA absorbs appreciably at $280 \mathrm{~nm}$ giving a broad fluorescence with maximum at around $340-350 \mathrm{~nm}$. The molecule AODIQ does not absorb significantly at $280 \mathrm{~nm}$ but absorbs appreciably at $350 \mathrm{~nm}$. The fluorescence of AODIQ in HSA environment has a maximum at $488 \mathrm{~nm}$. Considering the fluorescence band of tryptophan and the absorption band of AODIQ, the pair was judged a suitable donor-acceptor system for the FRET study. On gradual addition of AODIQ, the fluorescence intensity of the tryptophan residue present in the HSA decreased with concomitant increase in the fluorescence intensity of AODIQ through an isoemissive point at $438 \mathrm{~nm}$ (figure 4).

Figure 4 reflects an efficient energy transfer from the tryptophan group residue present in HSA to AODIQ. That the complexation process is effective even in low concentration of HSA is reflected in the linearity of the Benesi-Hildebrand double reciprocal plot (figure 3) suggesting that the stoichiometry of the probe-HSA complex is $1: 1$. It is important to mention here that we observed neither any additional absorption band for the mixture of HSA and

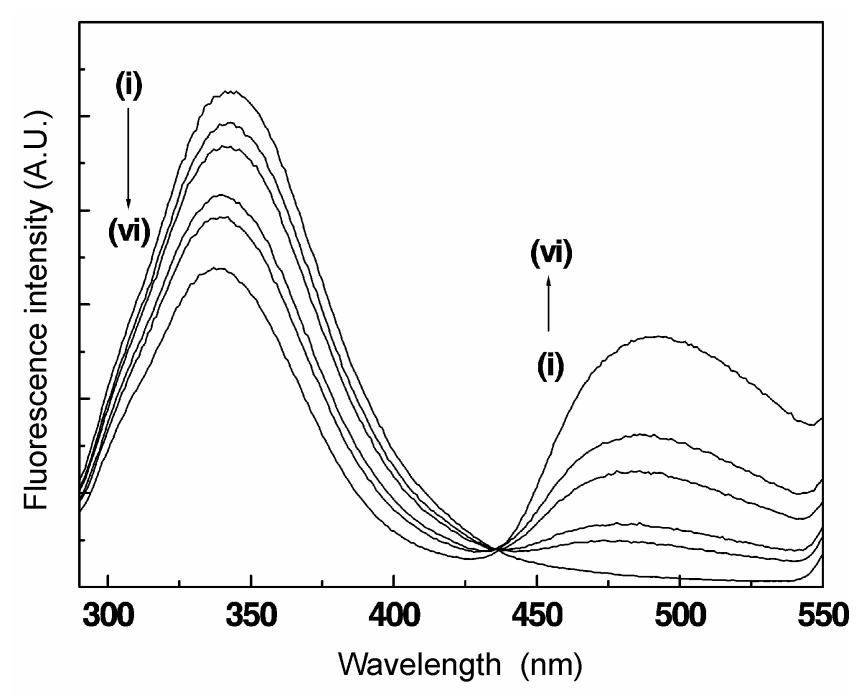

Figure 4. Fluorescence spectra of HSA as a function of AODIQ concentrations $\left(\lambda_{\mathrm{exc}}=280 \mathrm{~nm}\right)$ in aqueous HEPES buffer solution. Curves (i) $\rightarrow$ (vi) correspond to $0,4 \cdot 4,6 \cdot 5,13 \cdot 1,17 \cdot 4,21 \cdot 8 \mu \mathrm{M}$ AODIQ concentrations. 
AODIQ nor any deformity in the spectral pattern of the HSA-AODIQ system from that of the resultant of the individual absorption spectra corresponding to the donor and the acceptor taking into consideration the composition of the components in the mixture. Furthermore, the fluorescence spectrum of the mixture of donor and acceptor did not show any additional new band other than the individual emission bands of the two components. These factors taken together negate the formation of any permanent ground-state complex between the donor-acceptor pair in the solution ${ }^{25-27}$ as well as the formation of exciplex between the excited donor and the unexcited acceptor molecules. Thus the decrease in the fluorescence intensity of the donor with increasing acceptor concentration indicates a non-radiative energy transfer between the excited donor and the unexcited acceptor. ${ }^{26,27}$ Furthermore, the excitation profile monitoring the emission of AODIQ (at $488 \mathrm{~nm}$ ) shows that in the presence of HSA, besides the $S_{0} \rightarrow S_{1}$ transition of AODIQ ( $\lambda_{\mathrm{exc}}^{\max } \approx 420 \mathrm{~nm}$ ), a band with $\lambda_{\mathrm{exc}}^{\max } \approx 280 \mathrm{~nm}$ (corresponding to the tryptophan) appears. This provides strong evidence for the occurrence of Förster-type resonance energy transfer from the tryptophan moiety (donor) present in HSA to the AODIQ molecule (acceptor). ${ }^{21,27-29}$ Consistent with the work of Lakowicz, ${ }^{1}$ Sengupta et $a l^{30,31}$ De $e t a l^{27}$ and our own laboratory, ${ }^{20,32}$ we assign the observation to an efficient Förster-type resonance energy transfer from the tryptophan moiety to the AODIQ system.

The quenching of the steady-state fluorescence of the donor (tryptophan) with the addition of acceptor (AODIQ) was followed by the Stern-Volmer equation:

$$
F_{0} / F=1+K_{\mathrm{SV}}[Q],
$$

where $F_{0}, F$ are the fluorescence intensities of the donor in the absence and in the presence of the acceptor molecule, $[Q]$ is the acceptor concentration, and $K_{\mathrm{SV}}$ is the Stern-Volmer constant for the fluorescence quenching.

Figure 5 reveals that the Stern-Volmer plot is linear throughout the concentration range of the acceptor studied. The linearity of this plot proposes a single type of quenching process. ${ }^{1}$ The slope of the plot in figure 5 gives the Stern-Volmer constant $\left(K_{\mathrm{SV}}\right)$ as $1.9 \times 10^{4} \mathrm{~mol}^{-1} \mathrm{dm}^{3}$. Very good agreement between the two determined values, unequivocally confirms that $K_{\mathrm{SV}}$ is nothing but the binding constant $(K)$ itself and concludes that the fluorescence quenching results only from FRET. This further suggests that the donor (tryptophan) and the acceptor (AODIQ) are bound to the same protein at different locations but within quenching distance.

\subsection{Energy-transfer efficiency}

After establishing the occurrence of the FRET process in the HSA-AODIQ system, we intend to study the efficiency of the energy-transfer process between the two participating partners. Since AODIQ is a potential candidate for PDT application, determination of the efficiency of the energy-transfer process gains importance. We have also determined the distance between the donor and the acceptor though there is an uncertainty in the distance measurement, arising principally due to the orientation factor, which is a major problem in exploiting this technique. Several methods have been proposed to overcome this problem. ${ }^{33,34}$ According to Förster's non-radiative energy-transfer theory, ${ }^{35,36}$ the energy-transfer efficiency $E$ depends not only on the distance $\left(r_{0}\right)$ between the donor and the acceptor, but also on the critical energy transfer distance $\left(R_{0}\right)$ expressed by the following relation,

$$
E=R_{0}^{6} /\left(R_{0}^{6}+r_{0}^{6}\right),
$$

where $R_{0}$ is the characteristic distance, called Förster's distance or critical distance, at which the efficiency of transfer is $50 \%$. The magnitude of $R_{0}$ is dependent on the spectral properties of the donor and the acceptor molecules. $R_{0}$ is expressed as follows

$$
R_{0}^{6}=8.8 \times 10^{23}\left[\kappa^{2} n^{-4} \phi_{D} J(\lambda)\right] \text { in } \AA^{6},
$$

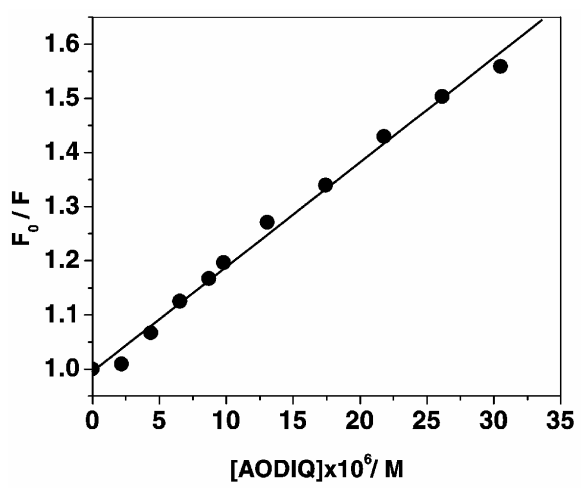

Figure 5. Stern-Volmer plot for the quenching of fluorescence of HSA by AODIQ in HEPES buffer solution. 
where $\kappa^{2}$ is the factor expressing the relative orientation of the donor to the acceptor molecule, $n$ is the refractive index of the medium, $\phi_{D}$ is the quantum yield of the donor $(0 \cdot 14)^{2}$ in the absence of the acceptor and $J(\lambda)$ is the overlap integral of the fluorescence emission spectrum of the donor and the absorption spectrum of the acceptor in units of $\mathrm{M}^{-1} \mathrm{~cm}^{3}$. The spectral overlap integral $J(\lambda)$ was calculated by numerical integration method. The overlap integral $J(\lambda)$ for a donor-acceptor pair is defined as, ${ }^{1,27,37}$

$$
J(\lambda)=\int_{0}^{\infty} F_{D}(\lambda) \varepsilon_{A}(\lambda) \lambda^{4} \mathrm{~d} \lambda
$$

where $F_{D}(\lambda)$ is the corrected fluorescence intensity of the donor at wavelengths $\lambda$ to $(\lambda+\Delta \lambda)$, with the total intensity normalized to unity and $\varepsilon_{A}(\lambda)$, the molar extinction coefficient of the acceptor at wavelength $\lambda$. The overlap spectrum is shown in figure 6 .

Förster's distance $\left(R_{0}\right)$ has been calculated assuming random orientation of the donor and acceptor molecules. Thus, from (4), we have calculated $R_{0}$ to be $73.6 \AA$ taking $\kappa^{2}=2 / 3, n=1.333$ (water), $\phi_{D}=$ $0 \cdot 14$. $^{2}$

The excellent correlation between the determined values of Stern-Volmer constant $\left(K_{\mathrm{SV}}\right)$ and binding constant $(K)$ indicates that the energy transfer efficiency $(E)$ between the two interacting partners in the present case is $\approx 100 \%$. The dimension of HSA, a $66 \mathrm{kD}$ protein may not be more than $20 \AA$. Considering this value for $r_{0}, 74 \AA$ for $R_{0}$ as estimated above and using (3), we get an estimate of $E$ as 0.9996 . This is consistent with the agreement between the

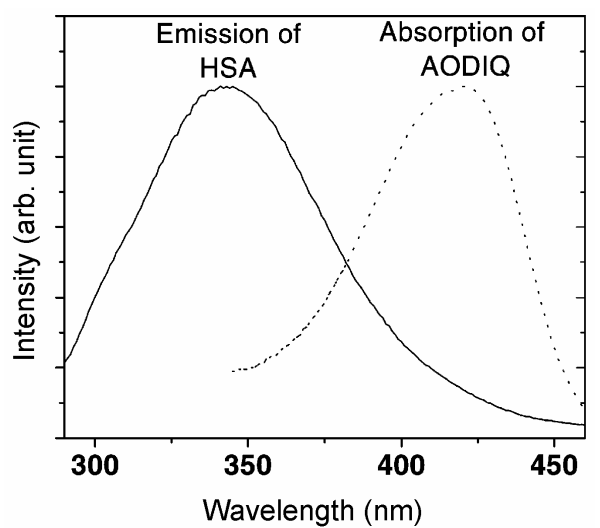

Figure 6. Overlap of fluorescence spectrum of HSA (solid line) and absorption spectrum of AODIQ (dotted line). values of $K_{S V}$ and $K$. This implies that the donor (tryptophan part of the HSA) and the acceptor (AODIQ) are bound to the same protein at different locations but within quenching distance.

\section{Conclusion}

The present photophysical study of HSA and AODIQ system in buffered aqueous environment leads to the following propositions. There is interaction between the probe and the albumin protein. A probe-HSA complex is formed with a $1: 1$ stoichiometry. Formation of the probe-HSA complex leads to efficient energy transfer from the tryptophan moiety of HSA to AODIQ. The quenching of the donor (HSA) fluorescence in the presence of the acceptor (AODIQ) is assigned completely to the FRET process.

\section{Acknowledgements}

Financial support from the Council of Scientific and Industrial Research (CSIR) and Department of Science and Technology, Government of India, is gratefully acknowledged. PD thanks CSIR for a fellowship. The authors sincerely thank one of the reviewers for their valued comments to upgrade the manuscript.

\section{References}

1. Lakowicz J R 1999 Principles of fluorescence spectroscopy (New York: Plenum)

2. Sytnik A and Litvvinyuk I 1996 Proc. Natl. Acad. Sci. USA 9312959

3. Szöõsi J, Damjanovich S, Mulhern S A and Trón L 1987 Prog. Biophys. Mol. Biol. 4965

4. Naik D B, Moorthy P N and Priyadarshini K I 1990 Chem. Phys. Lett. 168533

5. Becirra M A S, Ferreira S T, Strasser R J, Rodriguz W G, Beltrain C and Phyou A G 1999 Biochemistry 35 15925

6. Wojtuszewski K and Mukerji I 2003 Biochemistry 42 3096

7. Min H X and Carter D C 1992 Nature (London) 358 209

8. Peters T 1985 Serum albumin, advances in protein chemistry (New York: Academic Press) vol. 37, p. 161

9. Varela A P, Burrows H D, Douglas P and Miguel M G 2001 J. Photochem. Photobiol. A146 29

10. Tapia M J, Reyman D, Vinas M H, Carcedo C and Camecho J J 2002 Phys. Chem. Chem. Phys. 43676

11. Stolc S 1999 Life Sci. 651943

12. Dias A, Varela A P, Miguel M G, Maçanita A L, Becker R S and Burrows H D 1996 J. Phys. Chem. 10017970 
13. Varela A P, Miguel M G, Maçanita A L, Becker R S and Burrows H D 1995 J. Phys. Chem. 9916093

14. Mallick A and Chattopadhyay N 2005 Photochem. Photobiol. 81419

15. Mallick A, Haldar B and Chattopadhyay N $2005 \mathrm{~J}$. Photochem. Photobiol. B78 215

16. Chattopadhyay N, Dutta R and Chowdhury M $1989 \mathrm{~J}$. Photochem. Photobiol. A47 249

17. Giri V S, Maiti B C and Pakrashi S C 1984 Heterocycles 22233

18. Mallick A, Haldar B, Maiti S and Chattopadhyay N 2004 J. Colloid. Interface Sci. 278215

19. Mallick A, Haldar B and Chattopadhyay N $2005 \mathrm{~J}$. Phys. Chem. B109 14683

20. Mallick A, Haldar B, Sengupta S and Chattopadhyay N 2006 J. Luminescence 118165

21. Mallick A, Haldar B, Maiti S, Bera S C and Chattopadhyay N 2005 J. Phys. Chem. B109 14675

22. Mallick A, Bera S C, Maiti S and Chattopadhyay N 2004 Biophys. Chem. 1129

23. Mallick A, Maiti S, Haldar B, Purkayastha P and Chattopadhyay N 2003 Chem. Phys. Lett. 371688

24. Benesi M L and Hildebrand J H $1949 \mathrm{~J}$. Am. Chem. Soc. 712703
25. Kozyra K A, Heldt J R, Diehl H A and Heldt J 2002 J. Photochem. Photobiol. A152 199

26. Azim S A, Ghazy R, Shaheen M and El-Mekawey F 2000 J. Photochem. Photobiol. A133 185

27. De S and Girigoswami A 2004 J. Colloid. Interface Sci. 271485

28. Sengupta B and Sengupta P K 2002 Biochim. Biophys. Res. Commun. 299400

29. Sengupta B and Sengupta P K 2003 Biopolymers 72 427

30. Sengupta B and Sengupta P K 2000 Biochem. Biophys. Res. Commun. 27713

31. Guharay J, Sengupta B and Sengupta P K 2000 Proteins: Struct., Function Genet. $\mathbf{4 3} 75$

32. Das P, Mallick A, Haldar B, Purkayastha P and Chattopadhyay N 2007 J. Mol. Liq. 13048

33. Domanov Y A and Gorbenko G P 2002 Biophys. Chem. 99143

34. Sklor L A, Hudson B S and Simoni R D 1977 Biochemistry 165100

35. van der Meer B W 2002 Rev. Mol. Biotechnol. 82181

36. Förster T 1948 Ann. Phys. 43755

37. Seth D, Chakraborty A, Setua P, Chakrabarty D and Sarkar N 2005 J. Phys. Chem. B109 12080 